\title{
THE PRINCIPLE OF LEAST INTERACTION ACTION
}

\section{W. OVENDEN}

Dept. of Geophysics and Astronomy, and Institute of Astronomy and Space Science, University of British Columbia, Vancouver 8, B.C., Canada

\begin{abstract}
The intuitive notion that a satellite system will change its configuration rapidly when the satellites come close together, and slowly when they are far apart, is generalized to 'The Principle of Least Interaction Action', viz. that such a system will most often be found in a configuration for which the time-mean of the action associated with the mutual interaction of the satellites is a minimum. The principle has been confirmed by numerical integration of simulated systems with large relative masses. The principle lead to the correct prediction of the preference, in the solar system, for nearly-commensurable periods. Approximate methods for calculating the evolution of an actual satellite system over periods $\sim 10^{9} \mathrm{yr}$ show that the satellite system of Uranus, the five major satellites of Jupiter, and the five planets of Barnard's star recently discovered, are all found very close to their respective minimum interaction distributions. Applied to the planetary system of the Sun, the principle requires that there was once a planet of mass $\sim 90 M_{\oplus}$ in the asteroid belt, which 'disappeared' relatively recently in the history of the solar system.
\end{abstract}

\section{References}

Ovenden, M. W.: 1973, in B. D. Tapley and V. Szebehely (eds.) Recent Advances in Dynamical Astronomy, D. Reidel Publ. Co., Dordrecht, p. 319.

Ovenden, M. W.: 1974, 'Bode's Law - Truth or Consequences', Vistas in Astronomy 16, in press. Ovenden, M. W., Feagin, T., and Graf, O.: 1974, Celes. Mech. 8, 455. 